\title{
Physical Activity and Obesity: Biomechanical and Physiological Key Concepts
}

\author{
Julie Nantel, ${ }^{1}$ Marie-Eve Mathieu, ${ }^{2,3}$ and François Prince ${ }^{3,4}$ \\ ${ }^{1}$ Department of Neurology and Neurological Sciences, Stanford University, Stanford, CA 94305, USA \\ ${ }^{2}$ CHU Sainte-Justine Research Center, Montreal, QC, Canada H3T 1C5 \\ ${ }^{3}$ Department of Kinesiology, University of Montreal, Montreal, QC, Canada H3C 3J7 \\ ${ }^{4}$ Marie-Enfant Rehabilitation Center, Montreal, QC, Canada H3T 1C5 \\ Correspondence should be addressed to Julie Nantel, nantelj@stanford.edu
}

Received 1 June 2010; Revised 16 August 2010; Accepted 30 September 2010

Academic Editor: Eric Doucet

Copyright ( $) 2011$ Julie Nantel et al. This is an open access article distributed under the Creative Commons Attribution License, which permits unrestricted use, distribution, and reproduction in any medium, provided the original work is properly cited.

Overweight (OW) and obesity (OB) are often associated with low levels of physical activity. Physical activity is recommended to reduce excess body weight, prevent body weight regain, and decrease the subsequent risks of developing metabolic and orthopedic conditions. However, the impact of $\mathrm{OW}$ and $\mathrm{OB}$ on motor function and daily living activities must be taken into account. OW and $\mathrm{OB}$ are associated with musculoskeletal structure changes, decreased mobility, modification of the gait pattern, and changes in the absolute and relative energy expenditures for a given activity. While changes in the gait pattern have been reported at the ankle, knee, and hip, modifications at the knee level might be the most challenging for articular integrity. This review of the literature combines concepts and aims to provide insights into the prescription of physical activity for this population. Topics covered include the repercussions of OW and OB on biomechanical and physiological responses associated with the musculoskeletal system and daily physical activity. Special attention is given to the effect of OW and OB in youth during postural (standing) and various locomotor (walking, running, and cycling) activities.

\section{Introduction}

Excess body weight and a low level of physical activity are closely linked. The 2004 Canadian Community Health Survey showed that obesity rates in adults were significantly higher in sedentary men (27\%) compared to both moderately active (17\%) and active individuals (20\%) [1]. The rates of obesity were also higher in sedentary (27\%) and moderately active women $(21 \%)$ than in active women (14\%) [1]. The Copenhagen City Heart Study also showed cross-sectionally that individuals with a high body mass index [(BMI, in $\mathrm{kg}$ of body mass $\times$ (height in $\left.\left.\mathrm{m})^{-2}\right]\right)$ are more sedentary than those with a lower BMI [2]. However, the longitudinal portion of the study, where 5142 individuals were evaluated every 5 years over a 15 -year period, revealed novel findings: (1) physical inactivity at one point in time was not associated with the subsequent development of obesity (OB) while (2) the development of OB was associated to the subsequent reduction in physical activity levels [2].
These findings apply to adults, and Shields [3] proposed, based on this cross-sectional study, that physical activity levels of children do not differ according to body weight status when the overall activity level is considered. Fulton et al. (2009) reported that physical activity of moderate to vigorous intensity correlates negatively with two adiposity indexes: $\mathrm{BMI}$ and fat mass index, representing $\mathrm{BMI} \times$ percent body fat $\times 100^{-1}$ [4]. Altogether, these findings indicate that age and physical activity type specificities are present in the relationship between physical activity and body weight status.

Inactivity as a potential cause and/or result of $\mathrm{OB}$ is of great interest considering the growing obesity rates. Worldwide, over 400 million adults were reported to be OB in 2005, and by 2015, more than 700 million individuals are expected to have this condition [5]. In Canada, OB has increased from 14 to $23 \%$ of adults in the last 25 years [1] and from 3 to $8 \%$ in children [3]. With such high numbers of $\mathrm{OB}$ individuals, it is crucial to understand 
these individuals' specific activity levels in order to plan and offer adapted interventions to counteract their expected reduction in physical activity. The beneficial effects would be to slow down, and even stop, the progression of weight gain in individuals with more severe classes of $\mathrm{OB}$ or to bring an $\mathrm{OB}$ individual into the overweight (OW) or normal weight (NW) range. Likewise, OB individuals could benefit from the cardiometabolic benefits of an active lifestyle to reduce their higher risks of diabetes, hypertension, and other cardiovascular diseases [1] as well as to reduce the risk of musculoskeletal conditions such as knee and hip osteoarthritis (OA) $[6,7]$.

The general purpose of this paper is to better understand the impact of OW and $\mathrm{OB}$ on motor activities, with a special focus on children. More specifically, Section 2 aims to present general information on structural and muscular particularities that need to be taken into account for OW and $\mathrm{OB}$ individuals. The effect of excess body weight on three major daily living activities is then presented in Sections 3 , 4 , and 5 , respectively, on standing, walking, and cycling. Biomechanical and physiological concepts will be presented for each activity. This structure will ease a more extensive understanding of the situation of $\mathrm{OW}$ and $\mathrm{OB}$ individuals performing these activities and could support the development of adapted interventions. The last section highlights concepts that deserve attention for program development and research areas that warrant consideration in a near future.

\section{Obesity and Musculoskeletal Disorders}

2.1. Structural Specificities. Overweight and obesity in adults lead to alterations of the musculoskeletal system that could put $\mathrm{OB}$ individual at higher risk of musculoskeletal pain [6-9]. Comparing OB (BMI $38.8 \pm 6.0 \mathrm{~kg} \times \mathrm{m}^{-2}$ ) and NW adults (BMI $24.3 \pm 3.0 \mathrm{~kg} \times \mathrm{m}^{-2}$ ) Hills et al. [8] reported that $\mathrm{OB}$ individuals had higher plantar pressure, especially under the longitudinal arch and on the metatarsal heads both when standing and walking. Other studies found a strong link between the BMI and knee OA [10-13]. In a large singleblinded, randomized control clinical trial, Messier et al. [6] showed that weight loss of about 5\% resulting from a combination of diet and physical exercise improved function and mobility and reduced pain in OW and OB adults with OA. However, both musculoskeletal pain and OA are midto long-term consequences of obesity and affect almost exclusively adults.

Studies in children and adolescents also showed an effect of OW on both foot structure $[14,15]$ and the plantar pressure distribution [16] that could lead $\mathrm{OW}$ and $\mathrm{OB}$ children to be more likely to experience foot discomfort during weight-bearing activities [16]. In a study, Mickle et al. [14] compared the foot characteristics derived from footprints and reported lower plantar arch height in $\mathrm{OB}$ children (age $4.3 \pm 0.9$ years; BMI, $18.6 \pm 1.2 \mathrm{~kg} \times \mathrm{m}^{-2}$ ) compared to NW children (age $4.3 \pm 0.7$ years; BMI, $15.7 \pm$ $0.7 \mathrm{~kg} \times \mathrm{m}^{-2}$ ). The authors proposed that this difference could be due to structural modifications of the foot due to the excess of bodyweight and that is more likely to cause functional complications in the adulthood. However, a correlation with a more direct measurement of the foot structure such as a radiographic could have strengthened the results of this study.

OB individuals have also been shown to modify the force alignment and consequently the distribution of forces at the knee during weight bearing. This has led several researchers to link alterations in force distribution, particularly those associated with varus malalignment (the load-bearing axis is shifted inward, causing more stress and force on the medial compartment of the knee), to the development of OA in obese adults [17-23]. In a review, Wearing et al. [24] highlighted that it was still unclear whether varus malalignment was the consequence or the cause of knee OA. In the pediatric $\mathrm{OB}$ population, the knee has been reported to be a common site of pain [25], while Gushue et al. [26] reported that OB children have an abnormal knee load during walking and concluded that, in the long term, this modification in the gait pattern could increase the risk of developing knee OA. There is a lack of a longitudinal study to determine the exact role of $\mathrm{OB}$ during childhood and in the development of OA. However, malalignment of the lower limbs has been linked to an increase in musculoskeletal discomfort during walking in OW children [24, 25].

2.2. Muscles, Physical Function, and Energy Expenditure. The muscular system is a complementary component to consider. Zoico et al. [27] investigated the importance of muscle mass in 167 elderly women and found that functional limitations, assessed by questionnaires and strength measurements, are a key parameter linked to activity energy expenditure. This group was the first to show that a BMI greater than or equal to $30 \mathrm{~kg} \times \mathrm{m}^{-2}$ is significantly associated with a higher level of functional limitation; $65 \%$ of OB women reported at least one limitation as opposed to $38 \%$ of NW and $41 \%$ of OW women. Unfortunately, the dichotomization of the limitation status (at least one versus none) impedes the assessment of the magnitude of the limitation profile of individuals with at least one limitation in the OB, OW, and NW groups. A second finding of this study is that a low relative fat free mass (FFM), expressed as $\mathrm{kg}$ of FFM $\times$ body height $(\mathrm{m})^{-2}$, is not associated with more physical limitations per se, but rather that a low percentage of FFM significantly increases the odds of functional limitations [27]. Thus, the ratio of the FFM to the total body mass appears to be important in identifying individuals at higher risk of functional limitations, and to a greater extent than the amount of FFM relative to their height. In other words, individuals require sufficient FFM to perform activities with an enlarged body mass. In this study, no significant differences were noted in the functional limitations of sarcopenic individuals, who have by definition an extremely low lean body mass. This is further reinforced by the fact that the absolute amount of lean body mass, expressed by height, in $\mathrm{m}^{-2}$, is not a key factor of physical mobility in this study. However, Stenholm et al. [28] showed that in adults aged 65 and over, low muscle strength combined with $\mathrm{OB}$ is associated with a slower walking speed, a higher sedentary level, a more rapid decline in strength, and a higher rate of new disabilities inhibiting mobility. 
In fact, the combined effects of adiposity and strength are present in regard to physical abilities. Therefore, muscle qualities such as strength, endurance, and FFM should be considered, along with adiposity itself, in physical activity practice and the energy equilibrium of $\mathrm{OB}$ adults. While these studies were conducted in older adults, the association between BMI, FFM, muscle qualities, and physical function in children remains unknown. Currently, energy expenditure and body composition is the only acquired knowledge for that population. A unique study conducted in 836 youths confined to a metabolic chamber for 24 hours, the reference method, revealed that FFM is the single largest contributor not only to the total energy expenditure, but also to the sleeping and activity energy expenditure [29]. Interestingly, Aucouturier et al. [30] showed that the FFM is higher in $\mathrm{OB}$ children in comparison to NW children and that the power generated per unit of FFM is the same in both groups. Consequently, the FFM in OB individuals is present in greater amounts and in a similar quality, which can at least partially compensate for an increased body weight. Currently, it remains unknown whether FFM also contributes to the musculoskeletal integrity and physical function of youths, although its importance in increasing the resting and activity energy expenditure is known.

\section{The Impact of Excess Body Weight on Standing}

3.1. Biomechanical Profile. During childhood, postural stability is considered to be a major component of the child's development. Morphological changes due to growth interfere with postural stability and lead to high variability in balancing strategies in children less than 6 years old [31]. In light of the latter, could the morphological changes due to obesity modify postural balance in children? Goulding et al. [32] used the Equitest Sensory Organization Test (SOT) to compare $25 \mathrm{OW}$ and $47 \mathrm{NW}$ boys aged between 10 and 21 years. The SOT test assesses the contributions of vestibular, visual, and somatosensory system contribution to balance and consists of three conditions where the force plates are stationary (eyes open, eyes closed, sway referenced visual surround) and three conditions where the force plates move (sway referenced conditions: eyes open and eyes closed, and one condition with sway-referenced visual surround) [33]. Despite the fair to good reliability of the SOT in children [34], it failed to discriminate postural balance between the NW and the OB groups. This led the authors to conclude that postural imbalance in $\mathrm{OB}$ was rather due to an insufficient musculature for their weight, than to proprioception or sensory function disturbances. However, a difference in bipodal balance between these groups is most likely to be infraclinical and would consequently be better served by a more sensitive assessment such as that of posturography.

The authors also used the Bruininks-Oseretsky balance test scores. The Bruininks-Oseretsky subtest of balance consists of three tasks of static unipodal stance (on the floor and on a balance beam) with eyes open and eyes closed, as well as five tasks assessing dynamic balance using different walking conditions (walking on a line, walking forward on a balance beam, walking forward heel-to-toe on a line, walking forward heel-to-toe on a balance beam, and stepping over a stick on the balance beam) [32]. Their results showed that the OW subjects had lower balance scores than the NW subjects. Furthermore, the Bruininks-Oseretsky score was moderately correlated (negative correlation) with the BMI, body weight, percentage of fat mass, and total fat mass assessed by a dual energy X-ray absorptiometry (DEXA) scan. The postural balance difference between groups was more apparent during a challenging one-leg stance on a balance beam, with both eyes open and closed. Similar results have been reported by Deforche et al. [35] comparing 25 OW to $32 \mathrm{NW}$ children aged from $8-10$ years. The OW group could not hold a unilateral standing position on a balance beam for as long as the NW. The study also assessed dynamic postural task such as heel-to-toe walking and tandem walking. Despite the wider base of support used by the OW children during normal walking they were able to complete both narrow walking tasks. However, they performed the tandem walking at a slower walking speed than did the NW and completed fewer steps in the heel-totoe walking than the NW group, possibly to increase postural stability [35]. Taken together, these results highlighted that OW children might be at greater risk of postural instability than NW in activities in which a narrow base of support is required. However, postural instability might not be exclusively related to activities involving a narrow base of support and has also been reported during a sit-to-stand task with self-determined foot placement. The sit-to-stand task has previously been used to assess functional lower limb strength between $13 \mathrm{OB}$ children and $13 \mathrm{NW}$ children [36]. The OB children took more time to complete the weight transfer from the seated to the standing position compared to NW children. Similar results were reported by Deforche et al. [35], with OW children being slower than NW children when asked to achieve 5 consecutive sit-tostand repetitions. In a second sit-to-stand task in which the children had to rise from a seated position $30 \mathrm{~cm}$ above from the floor, OW children took twice the time to do the weight transfer compared to the NW group. Moreover, the trunk kinematics was different from that of the NW group which involved a backward motion to initiate the weight transfer and a greater sway velocity while standing. In addition to the possible difficulty in OW to control the large inertia of the trunk, the difference between groups could also be attributed to insufficient lower limb strength relative to their weight $[35,36]$.

However, differences between OB and NW children have been reported even in tasks that required minimal muscular strength such as quiet standing. Using force platforms, static posturography showed that $\mathrm{OW}$ and $\mathrm{OB}$ during growth could interfere with postural stability $[32,37]$. McGraw et al. [37] have compared postural control in $10 \mathrm{OB}$ and 10 non$\mathrm{OB}$ children aged between 8 and 10 years old during quiet standing. The study was designed to assess postural stability in normal and challenging foot and visual conditions. A normal side-by-side position and a tandem foot position were used, along with normal, conflicted vision and dark 
environments. In both NW and $\mathrm{OB}$ children, postural stability performance was decreased in conditions where vision and the base of support were challenged concurrently. However, in OB children, the tandem position and the visually challenged conditions decreased their postural stability, as determined by their larger center of pressure $(\mathrm{CoP})$ displacement in both the anterior-posterior and the medial-lateral directions. While McGraw et al. [37] found no difference between the groups in the quiet standing with eyes open conditions, Nantel et al. [38] reported on subjects of similar age, significantly larger $\mathrm{CoP}$ amplitudes, as well as higher CoP velocities in the medial-lateral direction in the OB group when compared to the NW group. This difference between results in these studies could be due to methodology used to assess quiet standing. Indeed, Nantel et al. [38] used trials of 120 seconds while McGraw et al. [37] recorded the signal for 26.5 seconds. However, this latter difference was not discussed by the authors.

Taken together these studies suggest that $\mathrm{OB}$ children may be disadvantaged when asked to stand still for a mid to long period of time and that they are more affected by visual conflict and foot placement than NW children. Nantel et al. [38] proposed that this instability in quiet standing could be exacerbated in dynamic conditions and may increase the risk of falling in OB children. To another extend, McGraw et al. [37] concluded that these differences could have an impact on $\mathrm{OB}$ children's confidence in participating in physical activities. However, the small sample size used in these studies make the conclusion difficult to extend to the children $\mathrm{OW}$ and $\mathrm{OB}$ population in general.

3.2. Physiological Characteristics. Energy expenditure is another parameter that differs in the standing position according to body weight status. Lafortuna et al. [39] reported that upright standing in sedentary $\mathrm{OB}$ women $(n=$ $15)$ requires significantly more energy than in sedentary NW women $(n=6)$. Based on their results, OB women consumed an additional $0.1 \mathrm{~L} \times \mathrm{min}^{-1}$ of oxygen when standing, which corresponds to $126 \mathrm{~kJ}$ per hour based on the assumption that a liter of oxygen reflects an expenditure of about $21 \mathrm{~kJ}$ [40]. Obese individuals would then use $5040 \mathrm{~kJ}$ more than NW individuals if both stand for a 40-hour period. In theory, standing for a moderate to long period of time could, however, be associated with a higher fatigue level in $\mathrm{OB}$ individuals, especially if they are in poor physical condition. In adults (12 men and 12 women) without excess body weight, standing while performing clerical work was associated with a net increase in energy expenditure of $17 \mathrm{~kJ} \times$ hour $^{-1}$ [41]. On the basis of Lafortuna et al's [39] study, this should be even higher in OB individuals. However, NW subjects who took part in the study mentioned that they would prefer to replace sitting on a chair with sitting on an exercise ball, a position that uses an equivalent energy expenditure of $17 \mathrm{~kJ} \times$ hour $^{-1}$, before standing. The postural and musculoskeletal impacts of this work position are, at the moment, unknown in OW and $\mathrm{OB}$ individuals. Further studies are warranted before recommendations for the prolonged used of an exercise ball at work are brought forward. The absence of studies conducted in children also limit extrapolation of these findings to this population as well as to active individuals since only sedentary individuals were selected [39] or the physical activity level of participants was not specified [41]. The principal limit to consider pertains to methodological perspective. Current studies have reported an energy expenditure measured in the last five minutes of a 20-minute task conducted in laboratory settings. It remains unknown whether posture would change and individuals would lean on external support after 20 minutes, and thus, energy expenditure would be modified.

\section{The Impact of Excess Body Weight on Walking}

4.1. Biomechanical Profile. In adults, an increased body weight leads to major modifications in the gait pattern. OW and OB individuals have been shown to walk with a shorter step length, lower cadence and velocity, a decrease in the duration of the simple support phase and an increased double support phase $[9,42]$. Kinematic adaptations, such as a reduction in the range of motion at the knee and ankle, have also been reported [9, 42, 43]. Most of these changes have been associated with an increased load at the knee and the development of OA [6, 7, 42, 44-47]. Messier et al. [7] reported a positive association between body mass and compressive forces resultant forces and the abductor moment at the knee in $\mathrm{OB}$ adults with $\mathrm{OA}$. Furthermore, a modest weight reduction of $9.8 \mathrm{~N}$ (less than $1 \mathrm{~kg}$ ) was associated with a reduction of about $40 \mathrm{~N}$ in both compressive and resultant forces as well as with a reduction in the knee abduction moment. Similarly, comparing $10 \mathrm{OB}$ adults with NW, Browning and Kram [44] showed that OB individuals had a peak knee extensor moment about 50\% higher than NW when walking at $1.5 \mathrm{~m}^{-1}$ while a slight reduction in the walking speed to $1.0 \mathrm{~m}^{-1}$ seduced the knee peak extensor moment by $43 \%$ in OB adults. However, $\mathrm{OB}$ individuals walking at $1.1 \mathrm{~m} \times \mathrm{s}^{-1}$ had the same knee extensor moment as NW individuals walking at $1.5 \mathrm{~m} \times \mathrm{s}^{-1}$. In contrast, DeVita and Hortobágyi [43] reported a strong inverse relationship between the BMI and knee torque in $\mathrm{OB}$ individuals. Indeed, the altered kinematic gait pattern observed in the OB group was associated with a decreased torque at the knee at a self-selected pace (slower than the NW subjects). They also reported a knee torque similar to that of the NW group when walking at the same pace in spite of their higher body mass. However, these results were difficult to compare with results from other studies since the authors reported their results without normalizing for the bodyweight. At this time, there is no consensus in the literature on whether these modifications are directly related to the changes in morphology and limb alignment or if they are an adaptation to reduce pain in the presence of $\mathrm{OA}$ or to increase dynamic postural stability. As proposed by Messier et al. [7], longitudinal studies are needed to assess the long term effects of OW and weight loss on the gait pattern and $\mathrm{OA}$ progression.

The spatiotemporal differences between NW and OW children are similar to those reported in adults. OW children have a longer gait cycle and stance phase duration as well as a reduced cadence and velocity compared to NW 
$[37,48]$. Using kinematic and kinetic analyses, Hills et al. [48] compared $10 \mathrm{NW}$ and $10 \mathrm{OW}$ children. They reported less flexion at the knee and hip, as well as a flatter foot pattern during weight acceptance and an external rotation of the foot during the entire gait cycle in the OW children compared to NW children. Using a similar methodology Schultz et al. [49] compared $10 \mathrm{OB}$ with $10 \mathrm{NW}$ children aged between 8 to 12 years old and showed no difference in the kinematics between their groups, while Gushue et al. [26] reported a lower peak knee flexion during the stance phase in OW children, similar to the results in adults [43]. Gushue et al. [26] have suggested that the lower knee flexion could be a compensation strategy to avoid increasing the extensor load due to the large increase in body mass. However, this strategy in the sagittal direction could interfere with the ability of children to control the abductor moment at the knee. Indeed, the abduction moment at the knee has been shown to be larger in OW children $[26,49]$. Both groups proposed that this alteration in the frontal plane of motion could have long-term orthopedic implications. However, this latter conclusion has not been yet verified longitudinally.

In addition to the possible long-term limitations, Hills et al. [48] concluded that the gait pattern adaptations in OB children could be used to reduce the dynamic instability caused by the large body mass. Furthermore, they have proposed that the difficulty $\mathrm{OB}$ children have in adapting to different walking speeds might be a disadvantage when participating in physical activities involving frequent speed changes. Looking at spatiotemporal and kinetic during a self-selected pace walking task, Nantel et al. [50] showed that $\mathrm{OB}$ children were mechanically less efficient than NW children. Indeed, during the stance phase, despite a large absorption of mechanical energy in the hip flexor muscles, OB children were less efficient than NW children in transferring mechanical energy within the hip flexor muscles from the stance phase to the swing phase. Their results showed that compared to NW children, OB children used more mechanical energy when walking at the same speed.

4.2. Physiological Characteristics. Butte et al. [29] showed with a room respiration calorimeter that the energy expenditure in $460 \mathrm{OW}$ children walking at $1.25 \mathrm{~m} \times \mathrm{s}^{-1}$ was significantly greater than in $376 \mathrm{NW}$ children, by about $5.25 \mathrm{~kJ} \times \mathrm{min}^{-1}$. This finding supports a good agreement in bioenergetics of walking using both physiological and biomechanical analysis in NW, OW, and OB individuals. However, it has also been shown in adults that the net cost of transportation $\left(\mathrm{J} \times \mathrm{kg}^{-1}\right.$ for a given distance) is similar for $\mathrm{OB}$ and NW individuals at low speeds, such as $1 \mathrm{~m} \times \mathrm{s}^{1}(0 \%$ incline $): 2.7 \mathrm{~J} \times \mathrm{kg}^{-1} \times \mathrm{stride}^{-1}$ in $\mathrm{OB}$ and $2.6 \mathrm{~J} \times \mathrm{kg}^{-1} \times$ stride $^{-1}$ in NW individuals [39]. The authors expressed values per stride because no differences were noted among the groups at a given speed and incline. This is concordant with another study that showed that the oxygen consumption per kg of FFM was similar in $\mathrm{OB}$, OW and NW girls for a walk at a similar speed $\left(1.1 \mathrm{~m}^{\circ} \mathrm{s}^{-1}\right)$ [51]. However, this finding should not be extended to higher speeds, at which dissociation in the net cost of transportation is noted.

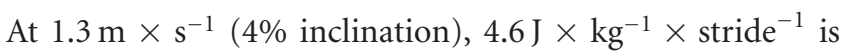
expended in $\mathrm{OB}$ youth and $4.1 \mathrm{~J} \times \mathrm{kg}^{-1} \times$ stride $^{-1}$ in $\mathrm{NW}$ youth, a significant difference [51]. This is similar to other results that show a higher discrepancy in the gross oxygen consumption $\left(\mathrm{L} \times \mathrm{min}^{-1}\right)$ between $\mathrm{OW}$ and NW young adults as speed increases: $53 \%$ difference at $0.5 \mathrm{~m} \times \mathrm{s}^{-1}$

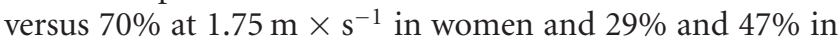
men for the same speeds [52].

With this higher energy expenditure at greater walking speeds, due in part to excess body weight and lower mechanical efficiency, it should not be a surprise that the absolute aerobic performance of $\mathrm{OB}$ youth is lower. Mastrangelo et al. [53] examined the running performance of $\mathrm{OB}$ children using a $1.6 \mathrm{~km}$ walk-run test and found that the running performance of OB children is lower. Significant differences were noted in terms of the body weight status when the minute oxygen consumption in $\mathrm{ml}$ per $\mathrm{kg}$ of body weight (48.3 in NW boys versus 41.6 in OB boys and 46.0 in NW girls versus 42.1 in $\mathrm{OB}$ girls) or the time to cover the distance (10 $\mathrm{min}, 34 \mathrm{sec}$ in NW boys versus $13 \mathrm{~min}, 8 \mathrm{sec}$ in OB boys and $13 \mathrm{~min}, 15 \mathrm{sec}$ in NW girls versus $14 \mathrm{~min}, 44 \mathrm{sec}$ in $\mathrm{OB}$ girls) composed the performance indicators.

Sometimes, the difference between the cardiorespiratory fitness of NW and OW children does not reach statistical significance [54]. However, the study of McGavock et al. [54] showed that a low oxygen consumption at baseline, expressed as $\mathrm{ml} \times \mathrm{kg}^{-1} \times \mathrm{min}^{-1}$, as assessed by a field test such as the Leger $20 \mathrm{~m}$ shuttle run, is predictive of a greater subsequent body weight increase and waist circumference. In fact, the risk of being classified as OW 12 months after the baseline evaluation was 3.5 times higher in children with low cardiorespiratory fitness (CRF) [54]. This appears to be an interesting tool in identifying children who do not have excess body weight yet but are at higher risk and might thus benefit from a primary prevention program. It is also similar to studies in adults conducted over more than 15 years in which a high CRF at baseline was associated with lower odds of obesity at followup [54]. Interestingly, McGavock et al. [54] showed that OW children tended to have a $75 \%$ reduced odds of remaining OW if their CRF was high at baseline (i.e., about $50 \mathrm{ml} \times \mathrm{kg}^{-1} \times \mathrm{min}^{-1}$ ). Therefore, a secondary prevention program to maintain a high CRF or increase the CRF in children with excess body weight appears justified. Studies conducted in Finland between 1976 and 2001 confirmed that the running performance of children had worsened over the years, but that the leisure time physical activity level and OB status explained a higher percentage of aerobic fitness in 2001 than in 1976 [55]. This can be encouraging because leisure time physical activity self-reporting is recently more popular among youth [56]. Despite this, it might not be sufficient to counteract the sedentary lifestyle outside organized sports. Also, the study indicates that more children are active during leisure time. However, a first limit of the study is the use of self-report assessment and a second limit is that the extent (frequency, duration, and intensity) of their physical activity in 1976 and 2001 was not monitored and could differ.

Structured physical activity programs can be beneficial to regulate body weight. The eight-month program conducted 
in Germany that includes behavioral and nutritional components reduced the disparities between $\mathrm{OB}$ children $(n=49$; 8-12 years old) and age-matched reference values for aerobic tests and some musculoskeletal tests where body weight can limit performance (e.g., push-ups) [57]. More specific interventions that help children with excess body weight overcome barriers associated with weight-bearing physical activities appear to be needed. A study conducted with OW girls revealed that, when compared to NW girls, they perceived equally that both peers and parents encouraged the practice of weight-bearing activities, which is good for their health, for a healthy outlook and for social relationships [58]. However, OW girls report more barriers to perform such activities, they find them to be less fun, they perceived themselves as performing poorly to a larger extent, and they have a lower self-efficacy toward the practice of weightbearing activities than NW girls. The study shows that even mothers of OW girls are aware that these activities are less fun for their daughters in a larger proportion than the mothers of NW girls. While it has been shown that barriers are higher in girls [59], it indicates that at least in girls, and potentially to a lower extent in boys, programs might address these important issues for both compliance to the intervention as well as persistence of an active lifestyle postintervention.

Several points must be taken into account regarding the CRF of $\mathrm{OB}$ children. First, a lower performance on a walk-run test, as characterized by a higher time to cover a given distance, a lower number of stages completed, a lower maximal speed, or a lower oxygen consumption expressed in $\mathrm{ml} \times \mathrm{kg}^{-1} \times \mathrm{min}^{-1}$ does not necessarily indicate a lower fitness profile. In fact, the absolute energy expenditure that $\mathrm{OB}$ individuals can generate can be higher despite a lower performance in terms of time or stages. An estimate of the energy deployed by the individuals can be estimated by multiplying the common CRF indicator ( $\mathrm{ml}$ of oxygen consumed $\times \mathrm{kg}^{-1} \times \mathrm{min}^{-1}$ ) by $0.021 \mathrm{~kJ} \times \mathrm{ml}$ of oxygen consumed $^{-1}$, the mean energetic equivalent of oxygen, and by body weight, in $\mathrm{kg}$. If everything other than body weight is held equal, an individual of $40 \mathrm{~kg}$ who jogs at $8 \mathrm{~km} \times \mathrm{h}^{-1}$, which represent eight metabolic equivalents or $28 \mathrm{ml} \times \mathrm{kg}$ of body weight ${ }^{-1} \times \mathrm{min}^{-1}$, would expend $23.5 \mathrm{~kJ} \times \mathrm{min}^{-1}$. For a child $25 \%$ lighter (i.e., $30 \mathrm{~kg}$ ), the energy expenditure would be reduced by a quarter, leading to an energy expenditure of $17.6 \mathrm{~kJ}$ per minute of jogging at the same speed. Considering the lower mechanical efficiency with increased body weight, it is possible that the energy expenditure of the heavier child is even higher than what general guidelines of energy expenditure suggest. No compendium of physical activity taking body weight status into consideration is currently available and individual assessment is necessary for precise measurements. If such measures are performed, the oxidative capacity of FFM is a good indicator of muscle quality that could also be measured. In order to appreciate this parameter, oxygen consumption can be reported per $\mathrm{kg}$ of FFM, assessed by bioimpedance scales or a DXA scan, for example. Then, more information on the abilities of muscles to perform cardiovascular work is available.

The measurement of ambulatory activities represents another important challenge from both the evaluation and intervention point of view. Pedometers are affordable devices that individuals can wear to provide objective measures of physical activity levels. Tudor-Locke et al. [60] reported that 6 - to 12-year-old girls and boys should take a minimum of 12,000 and 15,000 steps per day, respectively, to avoid OB. A major concern, however, is the accuracy of the step count with an increased body weight. Comparative studies clearly show that four factors reduce the step count accuracy: the type of pedometer, a low ambulatory speed, a tilted position of the pedometer, and a high body weight [61]. Piezoelectric pedometers are more suited to all individuals, but especially for $\mathrm{OW}$ and $\mathrm{OB}$ individuals, than springlevered pedometers based on the technology in which a suspended horizontal lever arm moves in response to the hip's vertical accelerations [61]. In adults with a BMI greater than 25 , spring-leveled pedometers significantly underestimated actual steps at speeds of $54-94 \mathrm{~m} \times \mathrm{min}^{-1}$ by about $35 \%$, while a piezoelectric pedometer, the New Lifestyle NL-2000, underestimated actual steps by an average of only $7 \%$ at $54 \mathrm{~m}$ $\times \min ^{-1}(P<.05)$ and was within $3 \%$ at speeds of $67-107 \mathrm{~m}$ $\times \min ^{-1}[61]$. In children, the difference in the accuracy of steps recorded according to the type of pedometer was not conclusive [62]. Clearly, the accuracy is extremely low for walking speeds of children with or without excess body weight. However, overall measurements were more precise for NW than OW children, regardless of the type of pedometer used. Recently, the utility of accelerometers worn at the ankle compared to pedometers was justified for both NW and OW children because errors were considerably lower than with pedometers and no difference was noted according to the body weight statuses of the children [62]. Perhaps the difference in the ambulatory activities during normal conditions of NW and OW individuals would be less if measured with more precise devices, but that remains to be demonstrated.

\section{The Impact of Excess Body Weight on Cycling}

While walking and running are good physical activities to lose weight, they imply supporting body weight at each step. When an individual has an excess of body weight such locomotor activities are surely much difficult and may be associated with various musculoskeletal discomfort and or pain. An alternative is to look for nonweight bearing locomotor activities such as cycling. However, to our knowledge most studies looking at cycling in $\mathrm{OB}$ used a more physiological approach.

Obese youth expend more energy than non-OB individuals to perform activities in which the body weight is not supported. But what about energy expenditure when the body weight is supported? Studies addressing this question have been conducted with girls and women. A first study done with $\mathrm{OB}, \mathrm{OW}$, and NW girls indicates that the difference in energy expenditure during activities like cycling or riding a scooter are lower than that observed for walking [51]. Unfortunately in the latter study, the restriction to only female limits the extrapolation to all the population. Despite the small differences, studies conducted in $\mathrm{OB}$ women showed higher oxygen consumption 
$\left(\mathrm{L} \times \mathrm{min}^{-1}\right)$ for a given cycling intensity when compared to NW women, which indicates higher energy expenditure. This higher oxygen consumption and thus energetic output makes it more difficult for individuals with excess body weight to perform a given cycling activity. Aerobic capacity, reported per $\mathrm{kg}$ of body mass, is often used as discussed previously in this paper, and $\mathrm{OB}$ individuals have been shown to have a lower aerobic capacity [63]. This exacerbates the difficulty in performing cycling activities; the action of cycling at a given output represents a higher percentage of their maximal capacity. An important fact is that the sole action of cycling without resistance requires more energy in $\mathrm{OB}$ individuals, especially for high revolutions per minute. At 0 Watt of external resistance, the slope of the regression line between revolutions per minute and energy expenditure is greater for $\mathrm{OB}$ than NW individuals [64]. At 0 Watt and 60 revolutions per minute, a common cycling speed used in clinical settings for physical tests, $\mathrm{OB}$ individuals spend about $33 \%$ more energy, or an extra $50 \mathrm{~J} \times \mathrm{s}^{-1}$, than $\mathrm{NW}$ individuals. This clearly shows that the body weight support conferred by the bike does not cancel all the difficulty associated to excess body weight.

Experimentally induced weight gain or weight loss is very informative to better understand the bioenergetics of weight changes. Goldsmith et al. [65] induced in a controlled clinical environment either a $10 \%$ weight gain or a $10 \%$ weight loss in NW and OB individuals ( $n=30 ; 53 \%$ men) while maintaining a training regimen during the experiment. Mechanical efficiency and energy expenditure were measured on an ergometer bicycle both before and after weight changes. On one hand, they showed that muscle work efficiency increased by $15 \%$ at low intensity exercise (<25 Watts) after body weight loss, whereas muscle work efficiency was reduced by $25 \%$ at low intensity (10 Watts) after weight gain. On the other hand, no change in skeletal work efficiency was measured when cycling at 50 Watts and after weight loss or at 25 Watts and after weight gain. The impact of weight thus appears to be more important at low absolute intensities. In youth, Butte et al. [29] reported that cycling at 20 Watts (light intensity) and at various speeds with a mean of 57 Watts (moderate intensity) was more demanding in terms of energy needed for OW than NW individuals. In OW boys, light and moderate intensities required 3.8 and $2.9 \mathrm{~kJ} \times \mathrm{m}^{-1}$ more than in NW boys, while in OW girls, an additional 2.1 and $3.8 \mathrm{~kJ} \times \mathrm{m}^{-1}$ were required, respectively [29]. Based on the study by Goldsmith et al. [65], cycling for 60 minutes at 10 watts was associated with an energy expenditure of $613 \mathrm{~kJ}$ at baseline versus $798 \mathrm{~kJ}$ after a $10 \%$ body weight gain. Therefore, cycling without external resistance, an activity potentially overlooked due to its low intensity, appears to be of greater interest from the perspective of exercise prescription. Another consideration for exercise planning is that individuals who lose weight with effective interventions should either extend the duration or increase the intensity to maintain energy expenditure and avoid weight regain; the same 60 minutes of cycling at 10 watts will reduce energy expenditure from 714 to $596 \mathrm{~kJ}$ after a $10 \%$ weight loss [65].
At maximal exertion, similar [63] or higher [39] maximal oxygen consumption $\left(\mathrm{L} \times \mathrm{min}^{-1}\right)$ is obtained in $\mathrm{OB}$ individuals. Despite this, OB women generate less power, by 18 Watts on average than NW women due in part to lower mechanical efficiency [66]. Maximal oxygen consumption is however lower in $\mathrm{OB}$ individuals when expressed per $\mathrm{kg}$ of body mass, as found with submaximal intensities, while no difference is noted per kg of FFM [39]. Therefore, similar oxygen consumption per unit of FFM suggests that on an ergocycle, maximal muscle capacities are the same regardless of body weight status. Interestingly, reasons to terminate a maximal test differ according to body weight status. Musculoskeletal pain was far more commonly reported in $\mathrm{OB}$ than in NW women (19 versus 4\%, resp.) as a reason to terminate a maximal test performed on an ergocycle [66]. $\mathrm{OB}$ women also report that they end the test less frequently because of leg fatigue (16 versus 38\%). Together, these findings indicate that musculoskeletal pain is an important factor that limits the execution of a maximal effort.

\section{Considerations for Physical Activity Program Development and Research Perspectives}

Both OW and OB have been associated with changes in musculoskeletal structure and mobility. While changes in the gait pattern have been reported at the ankle, knee, and hip, modifications at the knee level might be the most challenging for articular integrity. Several studies have reported a knee overload in $\mathrm{OB}$ individuals when walking at a normal or fast speed and have highlighted a possible role in the development of OA. Consequently, a reduction in walking speed has been recommended to avoid musculoskeletal degeneration in $\mathrm{OB}$ adults. However, weight reduction was shown to reduce pain, improve mobility, and reduce the load at the knee in $\mathrm{OB}$ adults. A higher speed is one key parameter in weight reduction because of the associated higher energy expenditure, especially for individuals with a higher body weight status. Moreover, diet combined with a one-hour training program three times a week, including weight training and walking, was reported to maximize weight loss when compared to diet or exercise alone. In children, the higher mechanical energy expenditure while walking makes it a good exercise to lose weight. Higher intensity activities such as fast walking or activities with frequent speed changes should be proposed depending on the presence of pain or dynamic postural instability. These studies highlighted the complexity of physical activity prescription in $\mathrm{OB}$ populations, especially in the presence of postural instability, pain, or OA.

Activities in which the body weight is supported appear to be an alternative to high intensity activities with potentially fewer musculoskeletal constraints. To the best of our knowledge, no studies have documented the biomechanical parameters linked to OA and other disorders in OB individuals during cycling. Based on studies of individuals who were standing, walking or cycling, training on an elliptical trainer, in which the body weight is not necessarily supported by a device but where the individual stands and trains without the impact associated with walking or running, could be 
an interesting compromise. Again, this would need to be confirmed in further studies.

What emerges from current studies is that simple movements such as standing, walking at low speed and cycling without resistance or with very low resistance should not be neglected. They increase energy expenditure and could be part of a healthy lifestyle when included in training programs as active recovery. Building the confidence of $\mathrm{OB}$ individuals practicing physical activities, even if the intensity appears low, is important because some of them, potentially more girls, report low confidence in physical activity. Including how physical activity and the fitness profile are reported and interpreted could improve the compliance of an $\mathrm{OB}$ individual in physical activity. Addressing only the time to cover a distance or the frequency and intensity of activities performed without taking into account body weight and, thus, energy expenditure results in an underestimation of the actual work performed. For example, the ratio of total energy expenditure to basal energy expenditure in OW and NW children was reported to be similar for various activities including walking and cycling [29]. Of course, most OB individuals would benefit from an increase in their energy expenditure; a good integration of biomechanical and physiological specificities might help optimize energy expenditure and musculoskeletal integrity.

\section{Conclusion}

Obesity and OW are two conditions for which the impact on physical activities goes far beyond the important body fat accumulation. Major changes were noted for foot, knee, and hip structures and are associated with discomfort, pain, and illness. This can seriously impede daily physical activity level and limit the performance of $\mathrm{OB}$ and $\mathrm{OW}$ persons during fitness tests.

For exercise prescription, we have shown that not all activities present the same difficulties at different intensities. Cycling is more demanding at low intensity for OW and $O B$ individuals while ambulatory activities are more difficult at high intensities. Strategies like slowing the self-selected walking pace, shortening step length, or reducing resistance should be considered in exercise prescription for individuals with excess body weight, and this includes children. A good understanding of biomechanical and physiological profile is mandatory for safe testing and effective prescription of physical activity in $\mathrm{OW}$ and $\mathrm{OB}$ individuals. Future researches should look at the effect of varying biomechanical constraints (cadence, step length, inclination) and physiological demands (various intensities) on energetic expenditure to optimize training effect.

\section{Acknowledgment}

J. Nantel and M.E. Mathieu are both first authors.

\section{References}

[1] M. Tjepkema, "Adult obesity," Health Reports, vol. 17, no. 3, pp. 9-25, 2006.
[2] L. Petersen, P. Schnohr, and T. I. A. Sørensen, "Longitudinal study of the long-term relation between physical activity and obesity in adults," International Journal of Obesity, vol. 28, no. 1, pp. 105-112, 2004.

[3] M. Shields, "Overweight and obesity among children and youth," Health Reports, vol. 17, no. 3, pp. 27-42, 2006.

[4] J. E. Fulton, S. Dai, L. M. Steffen, J. A. Grunbaum, S. M. Shah, and D. R. Labarthe, "Physical activity, energy intake, sedentary behavior, and adiposity in youth," American Journal of Preventive Medicine, vol. 37, supplement 1, pp. S40-S49, 2009.

[5] World Health Organization : Global Database on Body Mass Index, http://apps.who.int/bmi/index.jsp.

[6] S. P. Messier, R. F. Loeser, G. D. Miller et al., "Exercise and dietary weight loss in overweight and obese older adults with knee osteoarthritis: the arthritis, diet, and activity promotion trial," Arthritis and Rheumatism, vol. 50, no. 5, pp. 1501-1510, 2004.

[7] S. P. Messier, D. J. Gutekunst, C. Davis, and P. DeVita, "Weight loss reduces knee-joint loads in overweight and obese older adults with knee osteoarthritis," Arthritis and Rheumatism, vol. 52, no. 7, pp. 2026-2032, 2005.

[8] A. P. Hills, E. M. Hennig, M. McDonald, and O. Bar-Or, "Plantar pressure differences between obese and non-obese adults: a biomechanical analysis," International Journal of Obesity, vol. 25, no. 11, pp. 1674-1679, 2001.

[9] S. P. Messier, "Osteoarthritis of the knee and associated factors of age and obesity: effects on gait," Medicine and Science in Sports and Exercise, vol. 26, no. 12, pp. 1446-1452, 1994.

[10] N. J. Manek, D. Hart, T. D. Spector, and A. J. MacGregor, "The association of body mass index and osteoarthritis of the knee joint: an examination of genetic and environmental influences," Arthritis and Rheumatism, vol. 48, no. 4, pp. 10241029, 2003.

[11] D. Coggon, I. Reading, P. Croft, M. McLaren, D. Barrett, and C. Cooper, "Knee osteoarthritis and obesity," International Journal of Obesity, vol. 25, no. 5, pp. 622-627, 2001.

[12] S. P. Messier, R. F. Loeser, M. N. Mitchell et al., "Exercise and weight loss in obese older adults with knee osteoarthritis: a preliminary study," Journal of the American Geriatrics Society, vol. 48, no. 9, pp. 1062-1072, 2000.

[13] C. Ding, F. Cicuttini, F. Scott, H. Cooley, and G. Jones, "Knee structural alteration and BMI: a cross-sectional study," Obesity Research, vol. 13, no. 2, pp. 350-361, 2005.

[14] K. J. Mickle, J. R. Steele, and B. J. Munro, "The feet of overweight and obese young children: are they flat or fat?" Obesity, vol. 14, no. 11, pp. 1949-1953, 2006.

[15] D. L. Riddiford-Harland, J. R. Steele, and L. H. Storlien, "Does obesity influence foot structure in prepubescent children?" International Journal of Obesity, vol. 24, no. 5, pp. 541-544, 2000.

[16] A. M. Dowling, J. R. Steele, and L. A. Baur, "What are the effects of obesity in children on plantar pressure distributions?" International Journal of Obesity, vol. 28, no. 11, pp. 1514-1519, 2004.

[17] J. L. Astephen and K. J. Deluzio, "Changes in frontal plane dynamics and the loading response phase of the gait cycle are characteristic of severe knee osteoarthritis application of a multidimensional analysis technique," Clinical Biomechanics, vol. 20, no. 2, pp. 209-217, 2005.

[18] A. Chang, K. Hayes, D. Dunlop et al., "Thrust during ambulation and the progression of knee osteoarthritis," Arthritis and Rheumatism, vol. 50, no. 12, pp. 3897-3903, 2004. 
[19] A. J. Baliunas, D. E. Hurwitz, A. B. Ryals et al., "Increased knee joint loads during walking are present in subjects with knee osteoarthritis," Osteoarthritis and Cartilage, vol. 10, no. 7, pp. 573-579, 2002.

[20] D. E. Hurwitz, A. B. Ryals, J. P. Case, J. A. Block, and T. P. Andriacchi, "The knee adduction moment during gait in subjects with knee osteoarthritis is more closely correlated with static alignment than radiographic disease severity, toe out angle and pain," Journal of Orthopaedic Research, vol. 20, no. 1, pp. 101-107, 2002.

[21] A. Mündermann, C. O. Dyrby, D. E. Hurwitz, L. Sharma, and T. P. Andriacchi, "Potential strategies to reduce medial compartment loading in patients with knee osteoarthritis of varying severity: reduced walking speed," Arthritis and Rheumatism, vol. 50, no. 4, pp. 1172-1178, 2004.

[22] T. Miyazaki, M. Wada, H. Kawahara, M. Sato, H. Baba, and S. Shimada, "Dynamic load at baseline can predict radiographic disease progression in medial compartment knee osteoarthritis," Annals of the Rheumatic Diseases, vol. 61, no. 7, pp. 617-622, 2002.

[23] L. Sharma, D. E. Hurwitz, E. J.-M. A. Thonar et al., "Knee adduction moment, serum hyaluronan level, and disease severity in medial tibiofemoral osteoarthritis," Arthritis and Rheumatism, vol. 41, no. 7, pp. 1233-1240, 1998.

[24] S. C. Wearing, E. M. Hennig, N. M. Byrne, J. R. Steele, and A. P. Hills, "Musculoskeletal disorders associated with obesity: a biomechanical perspective," Obesity Reviews, vol. 7, no. 3, pp. 239-250, 2006.

[25] E. D. Taylor, K. R. Theim, M. C. Mirch et al., "Orthopedic complications of overweight in children and adolescents," Pediatrics, vol. 117, no. 6, pp. 2167-2174, 2006.

[26] D. L. Gushue, J. Houck, and A. L. Lerner, "Effects of childhood obesity on three-dimensional knee joint biomechanics during walking," Journal of Pediatric Orthopaedics, vol. 25, no. 6, pp. 763-768, 2005.

[27] E. Zoico, V. Di Francesco, J. M. Guralnik et al., "Physical disability and muscular strength in relation to obesity and different body composition indexes in a sample of healthy elderly women," International Journal of Obesity, vol. 28, no. 2, pp. 234-241, 2004.

[28] S. Stenholm, D. Alley, S. Bandinelli et al., "The effect of obesity combined with low muscle strength on decline in mobility in older persons: results from the InCHIANTI Study," International Journal of Obesity, vol. 33, no. 6, pp. 635-644, 2009.

[29] N. F. Butte, M. R. Puyau, F. A. Vohra, A. L. Adolph, N. R. Mehta, and I. Zakeri, "Body size, body composition, and metabolic profile explain higher energy expenditure in overweight children," Journal of Nutrition, vol. 137, no. 12, pp. 2660-2667, 2007.

[30] J. Aucouturier, N. Lazaar, E. Doré, M. Meyer, S. Ratel, and P. Duché, "Cycling peak power in obese and lean 6- To 8-year-old girls and boys," Applied Physiology, Nutrition and Metabolism, vol. 32, no. 3, pp. 367-371, 2007.

[31] A. Shumway-Cook and M. H. Woollacott, "The growth of stability: postural control from a development perspective," Journal of Motor Behavior, vol. 17, no. 2, pp. 131-147, 1985.

[32] A. Goulding, I. E. Jones, R. W. Taylor, J. M. Piggot, and D. Taylor, "Dynamic and static tests of balance and postural sway in boys: effects of previous wrist bone fractures and high adiposity," Gait and Posture, vol. 17, no. 2, pp. 136-141, 2003.
[33] L. M. Nashner, F. O. Black, and C. Wall III, "Adaptation to altered support and visual conditions during stance: patients with vestibular deficits," Journal of Neuroscience, vol. 2, no. 5, pp. 536-544, 1982.

[34] E. Geldhof, G. Cardon, I. De Bourdeaudhuij et al., "Static and dynamic standing balance: test-retest reliability and reference values in 9 to 10 year old children," European Journal of Pediatrics, vol. 165, no. 11, pp. 779-786, 2006.

[35] B. I. Deforche, A. P. Hills, C. J. Worringham et al., "Balance and postural skills in normal-weight and overweight prepubertal boys," International Journal of Pediatric Obesity, vol. 4, no. 3, pp. 175-182, 2009.

[36] D. L. Riddiford-Harland, J. R. Steele, and L. A. Baur, "Upper and lower limb functionality: are these compromised in obese children?" International Journal of Pediatric Obesity, vol. 1, no. 1, pp. 42-49, 2006.

[37] B. McGraw, B. A. McClenaghan, H. G. Williams, J. Dickerson, and D. S. Ward, "Gait and postural stability in obese and nonobese prepubertal boys," Archives of Physical Medicine and Rehabilitation, vol. 81, no. 4, pp. 484-489, 2000.

[38] J. Nantel, H. Centomo, and P. Prince, "Postural control and postural mechanisms in obese and control children," in Proceedings of the International Society of Biomechanics, Cleveland, Ohio, USA, August 2005.

[39] C. L. Lafortuna, F. Agosti, R. Galli, C. Busti, S. Lazzer, and A. Sartorio, "The energetic and cardiovascular response to treadmill walking and cycle ergometer exercise in obese women," European Journal of Applied Physiology, vol. 103, no. 6, pp. 707-717, 2008.

[40] E. Jéquier, K. Acheson, and Y. Schutz, "Assessment of energy expenditure and fuel utilization in man," Annual Review of Nutrition, vol. 7, pp. 187-208, 1987.

[41] E. A. Beers, J. N. Roemmich, L. H. Epstein, and P. J. Horvath, "Increasing passive energy expenditure during clerical work," European Journal of Applied Physiology, vol. 103, no. 3, pp. 353-360, 2008.

[42] P. Spyropoulos, J. C. Pisciotta, K. N. Pavlou, M. A. Cairns, and S. R. Simon, "Biomechanical gait analysis in obese men," Archives of Physical Medicine and Rehabilitation, vol. 72, no. 13, pp. 1065-1070, 1991.

[43] P. DeVita and T. Hortobágyi, "Obesity is not associated with increased knee joint torque and power during level walking," Journal of Biomechanics, vol. 36, no. 9, pp. 1355-1362, 2003.

[44] R. C. Browning and R. Kram, "Effects of obesity on the biomechanics of walking at different speeds," Medicine and Science in Sports and Exercise, vol. 39, no. 9, pp. 1632-1641, 2007.

[45] M. A. Davis, W. H. Ettinger, and J. M. Neuhaus, "Obesity and osteoarthritis of the knee: evidence from the National Health and Nutrition Examination Survey (NHANES I)," Seminars in Arthritis and Rheumatism, vol. 20, supplement 3, pp. 34-41, 1991.

[46] D. T. Felson, "The epidemiology of knee osteoarthritis: results from the Framingham Osteoarthritis study," Seminars in Arthritis and Rheumatism, vol. 20, no. 3, pp. 42-50, 1991.

[47] T. D. Spector, D. J. Hart, and D. V. Doyle, "Incidence and progression of osteoarthritis in women with unilateral knee disease in the general population: the effect of obesity," Annals of the Rheumatic Diseases, vol. 53, no. 9, pp. 565-568, 1994.

[48] A. P. Hills and A. W. Parker, "Gait characteristics of obese prepubertal children: effects of diet and exercise on parameters," International Journal of Rehabilitation Research, vol. 14, no. 4, pp. 348-349, 1991. 
[49] S. P. Shultz, M. R. Sitler, R. T. Tierney, H. J. Hillstrom, and J. Song, "Effects of pediatric obesity on joint kinematics and kinetics during 2 walking cadences," Archives of Physical Medicine and Rehabilitation, vol. 90, no. 12, pp. 2146-2154, 2009.

[50] J. Nantel, M. Brochu, and F. Prince, "Locomotor strategies in obese and non-obese children," Obesity, vol. 14, no. 10, pp. 1789-1794, 2006.

[51] J. L. Aull, D. A. Rowe, R. C. Hickner, B. M. Malinauskas, and M. T. Mahar, "Energy expenditure of obese, overweight, and normal weight females during lifestyle physical activities," International Journal of Pediatric Obesity, vol. 3, no. 3, pp. 177185,2008 .

[52] R. C. Browning, E. A. Baker, J. A. Herron, and R. Kram, "Effects of obesity and sex on the energetic cost and preferred speed of walking," Journal of Applied Physiology, vol. 100, no. 2, pp. 390-398, 2006.

[53] M. A. Mastrangelo, E. C. Chaloupka, and P. Rattigan, "Cardiovascular fitness in obese versus nonobese 8-11-year-old boys and girls," Research Quarterly for Exercise and Sport, vol. 79, no. 3, pp. 356-362, 2008.

[54] J. M. McGavock, B. D. Torrance, K. A. McGuire, P. D. Wozny, and R. Z. Lewanczuk, "Cardiorespiratory fitness and the risk of overweight in youth: the healthy hearts longitudinal study of cardiometabolic health," Obesity, vol. 17, no. 9, pp. 18021807, 2009.

[55] P. R. T. Huotari, H. Nupponen, L. Laakso, and U. M. Kujala, "Secular trends in aerobic fitness performance in 13-18-yearold adolescents from 1976 to 2001," British Journal of Sports Medicine, vol. 44, no. 13, pp. 968-972, 2010.

[56] M. Westerståhl, M. Barnekow-Bergkvist, G. Hedberg, and E. Jansson, "Secular trends in sports: participation and attitudes among adolescents in Sweden from 1974 to 1995," Acta Paediatrica, International Journal of Paediatrics, vol. 92, no. 5, pp. 602-609, 2003.

[57] U. Korsten-Reck, T. Kaspar, K. Korsten et al., "Motor abilities and aerobic fitness of obese children," International Journal of Sports Medicine, vol. 28, no. 9, pp. 762-767, 2007.

[58] J. A. Fulkerson, S. A. French, M. Story, P. J. Hannan, D. Neumark-Sztainer, and J. H. Himes, "Weight-bearing physical activity among girls and mothers: relationships to girls' weight status," Obesity Research, vol. 12, no. 2, pp. 258-266, 2004.

[59] M. F. Zabinski, B. E. Saelens, R. I. Stein, H. A. HaydenWade, and D. E. Wilfley, "Overweight children's barriers to and support for physical activity," Obesity Research, vol. 11, no. 2, pp. 238-246, 2003.

[60] C. Tudor-Locke, R. P. Pangrazi, C. B. Corbin et al., "BMI-referenced standards for recommended pedometerdetermined steps/day in children," Preventive Medicine, vol. 38, no. 6, pp. 857-864, 2004.

[61] S. E. Crouter, P. L. Schneider, and D. R. Bassett Jr., "Springlevered versus piezo-electric pedometer accuracy in overweight and obese adults," Medicine and Science in Sports and Exercise, vol. 37, no. 10, pp. 1673-1679, 2005.

[62] N. Mitre, L. Lanningham-Foster, R. Foster, and J. A. Levine, "Pedometer accuracy for children: can we recommend them for our obese population?” Pediatrics, vol. 123, no. 1, pp. e127e131, 2009.

[63] D. Ofir, P. Laveneziana, K. A. Webb, and D. E. O'Donnell, "Ventilatory and perceptual responses to cycle exercise in obese women," Journal of Applied Physiology, vol. 102, no. 6, pp. 2217-2226, 2007.
[64] B. Anton-Kuchly, P. Roger, and P. Varene, "Determinants of increased energy cost of submaximal exercise in obese subjects," Journal of Applied Physiology, vol. 56, no. 1, pp. 1823, 1984.

[65] R. Goldsmith, D. R. Joanisse, D. Gallagher et al., "Effects of experimental weight perturbation on skeletal muscle work efficiency, fuel utilization, and biochemistry in human subjects," American Journal of Physiology, vol. 298, no. 1, pp. R79R88, 2010.

[66] M. Hulens, G. Vansant, R. Lysens, A. L. Claessens, and E. Muls, "Exercise capacity in lean versus obese women," Scandinavian Journal of Medicine and Science in Sports, vol. 11, no. 5, pp. 305-309, 2001. 


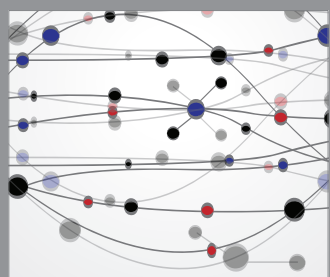

The Scientific World Journal
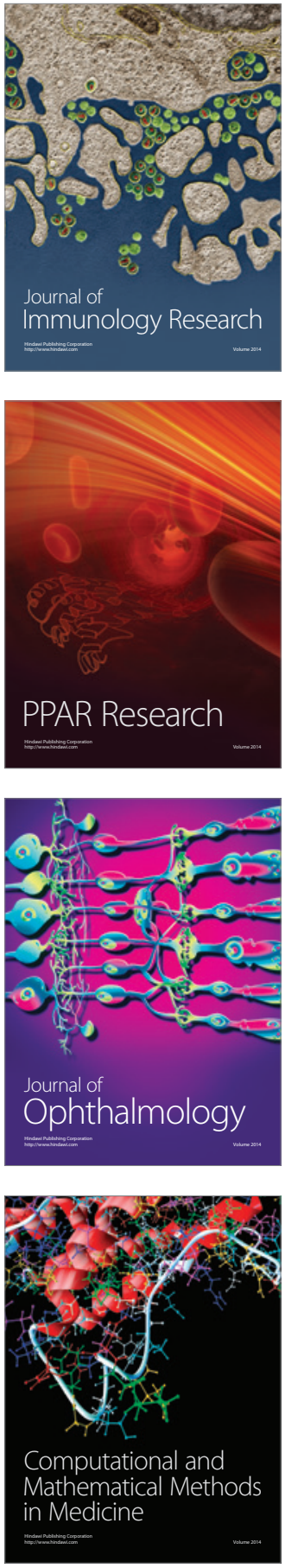

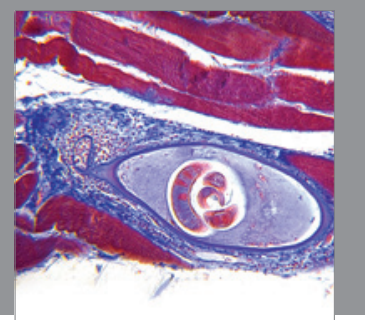

Gastroenterology

Research and Practice
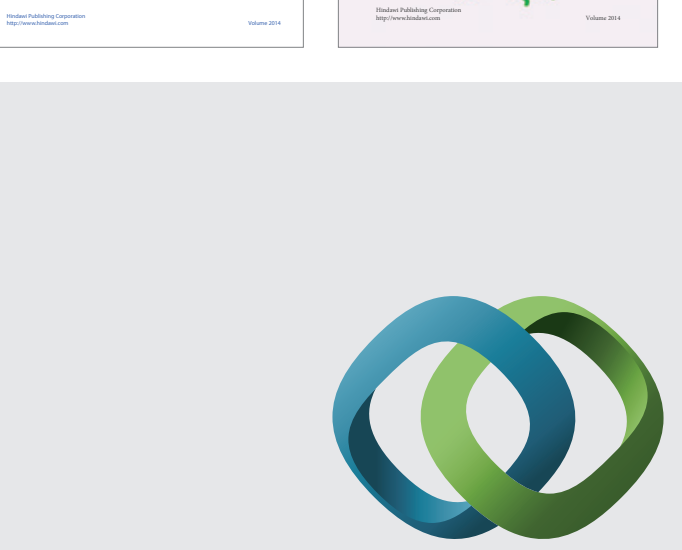

\section{Hindawi}

Submit your manuscripts at

http://www.hindawi.com
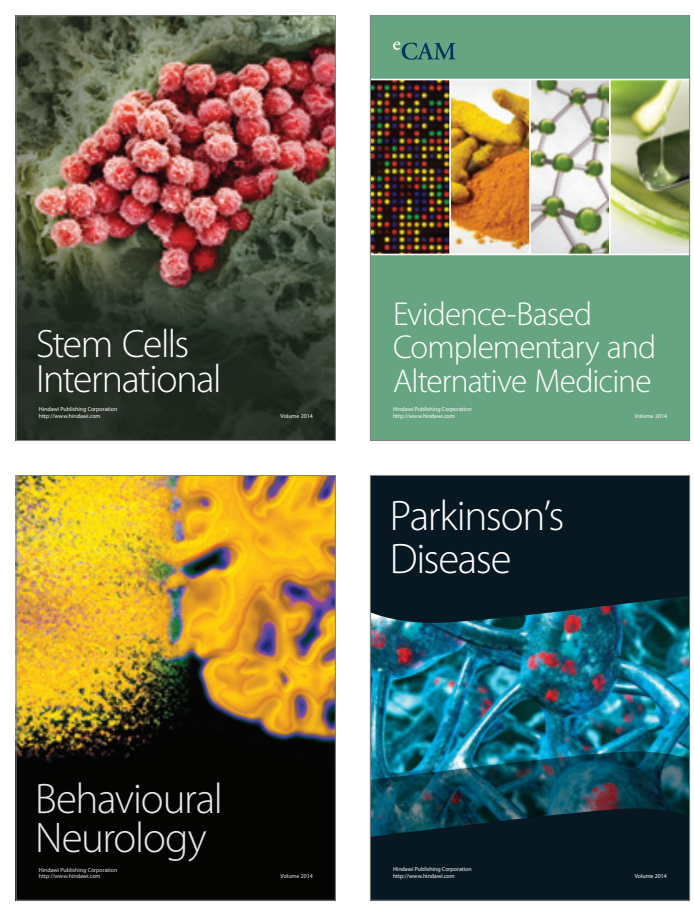

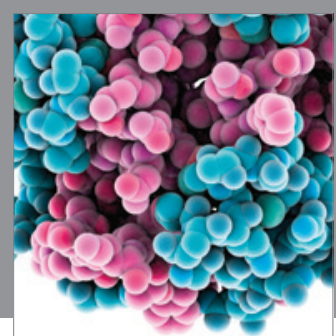

Journal of
Diabetes Research

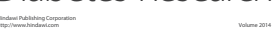

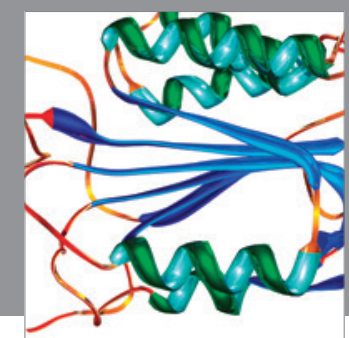

Disease Markers
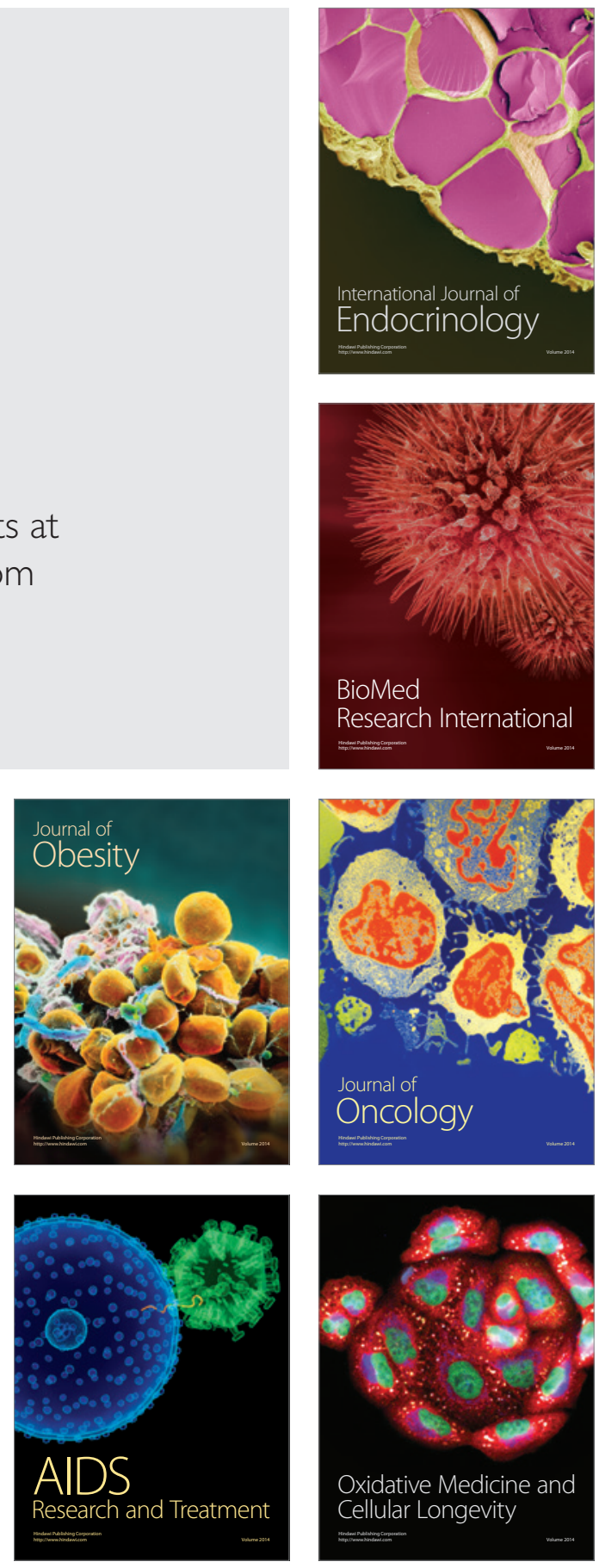